\title{
Team Whoosh Generator 2015 WSGC Collegiate Rocket Competition
}

\author{
James Ihrcke, Eric Johnson, Leeta Russell, Stephan Skibinski, Braden Thiel
}

Milwaukee School of Engineering

\begin{abstract}
The objectives of the 2015 Wisconsin Space Grant Consortium (WSGC) Collegiate Rocket Competition were to design and build a "boosted dart" high-powered rocket to allow the dart stage to reach the highest possible apogee and to conform to design restrictions and added design objectives. The rocket was required to use a Cesaroni I-445 motor as specified by WSGC, use a flight data recorder provided by WSGC, and be safely recovered in a flyable condition by use of a parachute system. The dart was required to be an unpowered upper stage that drag separates from the booster without imparting momentum onto the dart. In addition to the altimeters already on board, a noncommercial data acquisition system was required to record data on the rotation of the rocket in the $\mathrm{X}, \mathrm{Y}$, and $\mathrm{Z}$ axes over time. Finally, the rocket was required to record downward facing video of the flight starting at the time of launch.

Included in this report are design details considered, anticipated performance, photos of constructed components, and flight results.
\end{abstract}

Team Whoosh Generator thanks the Wisconsin Space Grant Consortium (WSGC) for the funding to make this project possible. Also thanks to this year's rocket shadows: Lucas Ferrandoquielz, Henry Moroder, Jordan Petrie, and Cameron Wilson for their help in the design and construction of this year's rocket. 


\subsection{Rocket Design and Construction}

The following subsections will detail the airframe design, nosecone and fin design, rocket stability, electronics bay design, the data acquisition system, camera system, pressure relief considerations, and recovery method.

1.1 Airframe design. The body tubes could be selected from several different kinds of materials, including cardboard, fiberglass, and PVC. Cardboard was selected as the body tube material for the rocket because of its simplicity, strength, price, and ease of cutting and drilling. Cardboard LOC body tubes have also proven to work well in the construction of previous years' rockets.

The rocket's diameter was determined after several brainstorming sessions. The final two designs were a dart diameter of 38 millimeters with a booster diameter of 54 millimeters or a dart diameter of 54 millimeters with a booster diameter of 3 inches. After weighing the pros and cons, the 54 millimeter dart, 3 inch booster combination was chosen because it was the minimum size required for the electronics to fit in the dart and still provide a large enough drag difference from dart to booster to cause drag separation upon motor burnout.

The body tube lengths were heavily dependent on the size of the components being placed inside of them. OpenRocket, a free open source rocket design software program, was the primary software used to design much of the rocket. OpenRocket was used to calculate the optimum body tube lengths. It was found that the booster section (that houses the motor mount and a parachute) would have a body tube length of 21.5 inches and a total length of 29 inches including transition and tail cone. The dart section (that houses a smaller parachute, the electronics bay, and the downward facing camera) would have a total body tube length of length of 23.75 inches and a total length of 38.25 inches including nose cone and tail cone. The total length of the rocket including the nose cone and the tail cone motor retainer was 64.61 inches.

1.2 Nose cones. With the body tube size of the dart chosen as a 54 millimeter LOC body tube, an ogive 54 millimeter PNC nose cone was selected to be used. The ogive shape was chosen because it has a low coefficient of drag which allows for an increased apogee. In order to provide a strong base for the dart and reduce drag, another nose cone was purchased to act as a tail cone. The tail cone was cut to a length of 5 inches to allow for the downward facing camera to be recessed in approximately 0.25 inches. The tail cone also provided a sturdy base for the attachment point of the dart to the booster and it reduced the pressure drag on the dart, increasing its apogee. For the transition on the booster, a 3 inch PNC ogive nose cone was used. The transition was cut to a length of 6 inches to allow the dart's tail cone to slide into it for alignment purposes. The dart needs to be aligned and held plumb with the rest of the rocket during the thrust phase. In order to do this, a custom bulkhead was created and epoxied inside of the booster transition cone which allowed the dart's tail cone to firmly fit inside of it yet still allow for easy separation.

1.3 Fins. The fins are the main component that determines the location for the center of pressure on a rocket and therefore the stability of the rocket. The fin design was determined by placing different shapes and sizes of fins in OpenRocket until a stable ratio between the center of gravity and center of pressure was obtained. The complete rocket was modeled and the dart and booster separately to ensure the rocket would stable throughout its entire flight. It was determined to use 3 fins spaced evenly around the rocket on both the booster and the dart. The fins chosen for the 
dart were A-08 G-10 prism fiberglass fins and for the booster were B-08 G-10 prism fiberglass fins from Public Missiles. The fins were attached to the booster by placing them through fin slots in the lower body tube and using epoxy to attach them to the motor mount tube on the outer and inner surfaces of the body tube. The fins were attached to the dart by epoxying them directly to the outside of the rocket. A photo of the booster and dart fins is shown in Figure 1.

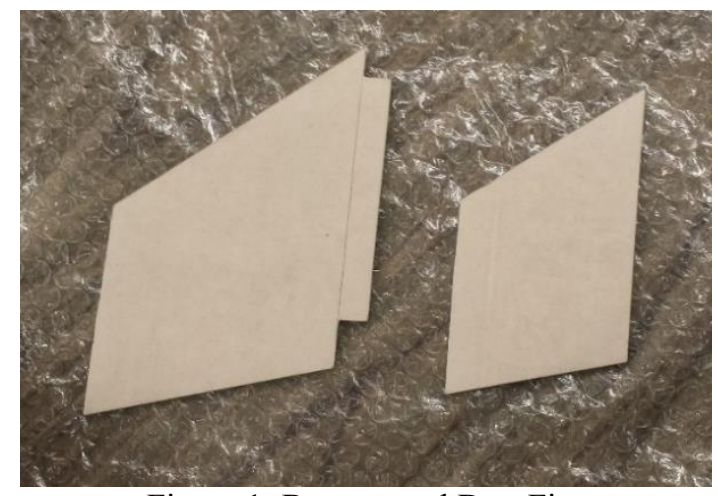

Figure 1: Booster and Dart Fins

1.4 Rocket stability. The relationship between the center of pressure $(\mathrm{CP})$ and center of gravity (CG) is one of the most important relationships in high-powered rocketry. The center of pressure is defined as the point at which aerodynamic forces on the rocket are centered. The center of gravity is the location at which the full weight of the rocket can be considered to act as a single force. The ratio between the locations relative to the rocket diameter can be used to predict the stability of the rocket during flight. Generally, the center of gravity must be at least one (but not more than two) body tube diameters in front of the center of pressure. The locations of the center of pressure and center of gravity were determined for this design using the OpenRocket software.

Table 1 shows the locations of the $\mathrm{CP}$ and $\mathrm{CG}$ and the caliber of stability for the full rocket at ignition and for the dart after separation according to the OpenRocket simulation.

Table 1: Locations of CP and CG (In Inches from Nose Cone Tip)
\begin{tabular}{|c|c|c|c|}
\hline & CP & CG & Stability (Caliber) \\
\hline Full Rocket at Ignition & 46.58 & 41.23 & 1.73 \\
\hline Dart after Separation & 25.31 & 21.62 & 1.63 \\
\hline
\end{tabular}

From this analysis, it can be concluded that both the dart and the booster will be stable during the entire ascent portion of the flight.

1.5 Electronics bay. To save on space in the dart, the dart's nose cone was cut open and a bulkhead was epoxied inside 3.5 inches aft of the tip of the cone with a threaded rod extending out. This allowed a fiberglass sled to be fed onto the rod and make use of the space in the nose cone that would otherwise be wasted. The nose cone was coupled with a 6.75 inch long 54 millimeter diameter body tube and a 4.75 inch long 2.14 inch diameter coupler tube. The coupler fit perfectly into the 54 millimeter airframe, joining the electronics bay with the rest of the dart. One barometric pressure altimeter was placed in the electronics bay, an ALTS25 which had been used in previous years. The altimeter was used to deploy the parachute in the dart as well as record 
the altitude of the rocket. The electronics bay also held an Arduino Uno with a 3 axis accelerometer that recorded roll data during the flight. Finally the electronics bay held the Raven 3 (WSGC flight data recorder) along with a 9 volt battery that powered the previously mentioned electronics. Two screw switches were placed in the electronics bay to allow easy arming of devices on the launch pad. One screw switch was for turning on the WSGC flight data recorder and Arduino and the other was for arming the altimeter. A terminal block was placed at the end of the bay to allow easier attachment of the black powder charge on launch day. The assembled electronics bay is shown in Figure 2.

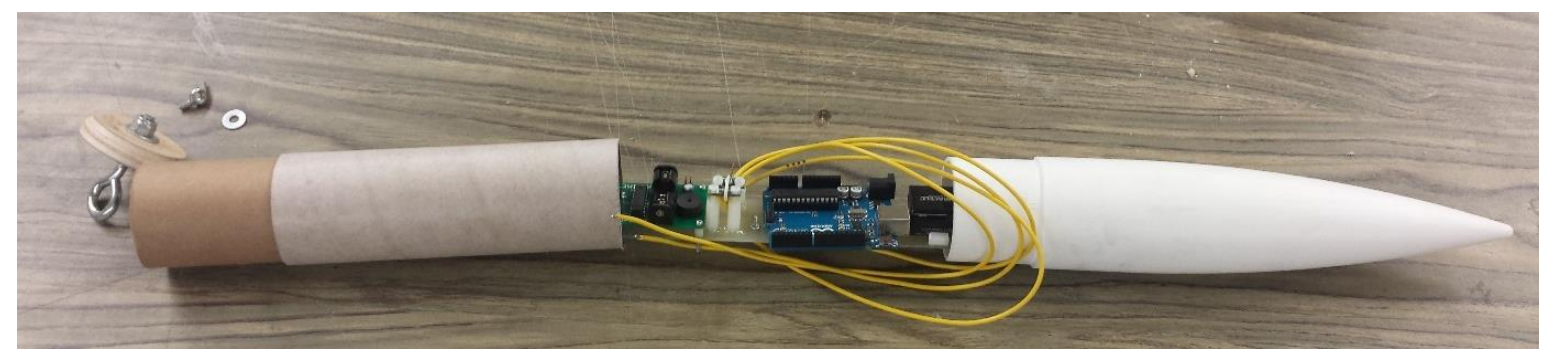

Figure 2: Electronics Bay Assembly

1.6 Data acquisition system (DAS). A requirement of this year's competition was to record flight data that could be used to determine the rotation of the dart during flight using a device other than an off-the-shelf pitch or roll data logger.

It was determined that a 3-axis accelerometer would be the most cost-effective and compact device that could be used to acquire both pitch and roll data. In an effort to utilize existing resources, the search for the right accelerometer was limited to those compatible with an Arduino Uno. After searching through a variety of accelerometer options, it was determined that the Analog Devices ADXL-335 three-axis accelerometer was best suited for our needs, shown in Figure 3.

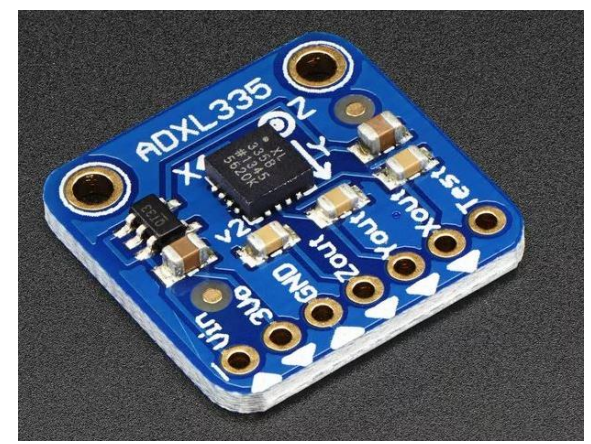

Figure 3: Three-axis Accelerometer

This three-axis accelerometer was available in two different breakout boards with either a digital interface or an analog interface. The analog interface was favored over the digital interface simply due to familiarity and the bypass of potential bit error when using i2c protocol. The output of the ADXL-335 consists of an analog voltage output with respect to each Cartesian linear acceleration, the output voltage ranging from 0 to 3.3 volts. The acceleration sensing capabilities of this accelerometer was listed as $\pm 3 \mathrm{~g}$ in the data sheet. Equation 1 was determined using the voltage and acceleration range of each axis.

$$
g_{n}=1.818 V_{n}-3
$$


After choosing the accelerometer, the logging of the three-axis data was addressed. Arduino Uno shields were evaluated to determine the best way to record data and extract it after flight. Several breakout boards were considered, each using some form of SD storage and digital interface to record data. It was determined that the Adafruit 1141 SD shield for the Arduino Uno was the best option, providing compact design and direct compatibility shown in Figure 4.

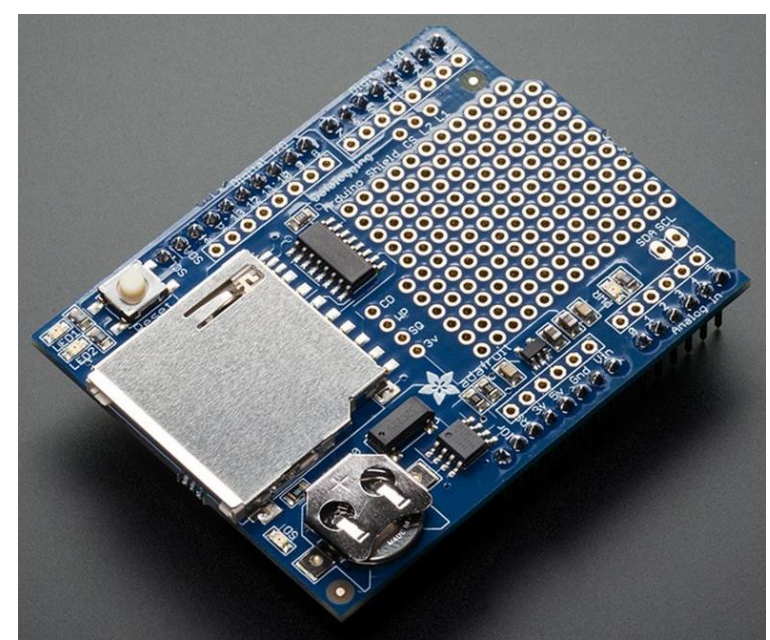

Figure 4: Adafruit 1141 SD shield for the Arduino Uno

To record data with the SD shield on the Uno, a data logging file was written in the Arduino code interface. This data logging file used three analog channels on the Uno to read each of the analog outputs of the accelerometer. These analog voltages were sampled at a rate of $100 \mathrm{~Hz}$ and saved into individual data files on the SD card for each of the three axes. The code was designed to start recording data after a short delay, allowing excess data to be left unrecorded while preparing the rocket on the launch pad.

The data stored on the SD card could be accessed using Microsoft Excel, opening a single column dataset for each accelerometer axis. The recorded values were analog voltages ranging from 0 to 3.3 volts. These data points were turned into points of linear acceleration using Equation 1. After converting each set of voltage data to acceleration, Equations 2 and 3 are used to calculate the desired pitch and roll characteristics needed to meet the flight requirements.

$$
\begin{gathered}
\text { pitch }=\arctan \left(\frac{G_{y}}{\sqrt{G_{x}^{2}+G_{z}^{2}}}\right) \\
\text { roll }=\arctan \left(-\frac{G_{x}}{G_{z}}\right)
\end{gathered}
$$

By using the combination of the Arduino Uno, SD shield, and three-axis accelerometer, a data logging setup was constructed. The Arduino Uno collected analog voltage data from the three-axis accelerometer representing the linear acceleration on each Cartesian axis. The firmware on the Arduino logs the analog signals at a sampling frequency of $100 \mathrm{~Hz}$ onto the SD card on the Arduino shield. The data extracted from the SD card was then used to model the roll in the rocket's flight in Microsoft Excel. 
1.8 Camera system. The competition required a downward facing camera to record the entirety of rocket's flight. Different action cameras were researched and compared against one another to determine which would work best. A decision matrix was created to make the comparison easy shown in Table 2.

Table 2: Decision Matrix of Possible Camera Choices.

\begin{tabular}{|c|c|c|c|c|c|c|c|c|c|}
\hline & \multicolumn{2}{|c|}{ Polaroid Cube } & \multicolumn{2}{|c|}{$\begin{array}{l}\text { Contour } \\
\text { ROAM } 2\end{array}$} & \multicolumn{2}{|c|}{$\begin{array}{l}\text { Mini Cylinder } \\
\text { Shaped Sport }\end{array}$} & \multicolumn{2}{|c|}{ Go Pro Hero } & \\
\hline & Rank* & Score & Rank* & Score & Rank* & Score & Rank* & Score & Weight \\
\hline Dimensions & 4 & 16 & 2 & 8 & 3 & 12 & 1 & 4 & $\mathrm{x} 4$ \\
\hline Price & 3 & 6 & 2 & 4 & 4 & 8 & 1 & 2 & $\mathrm{x} 2$ \\
\hline Weight & 2 & 4 & 1 & 2 & 4 & 8 & 3 & 6 & $\mathrm{x} 2$ \\
\hline Trusted Brand & 4 & 4 & 4 & 4 & 1 & 1 & 4 & 4 & $\mathrm{x} 1$ \\
\hline Video Quality & 1 & 1 & 1 & 1 & 1 & 1 & 1 & 1 & $\mathrm{x} 1$ \\
\hline View Angle & 2 & 2 & 4 & 4 & 1 & 1 & 3 & 3 & $\mathrm{x} 1$ \\
\hline $\begin{array}{c}\text { Weighted } \\
\text { Total } \\
\end{array}$ & & 33 & & 23 & & 31 & & 20 & \\
\hline
\end{tabular}

*Rank: 1 = least beneficial, $4=$ most beneficial

From the decision matrix it was shown that the Polaroid Cube would work the best. Its small cubed shape provided flexibility in the different directions that it could be mounted inside the body tube. Its price was also a plus because it was the second cheapest option and had a trusted brand name. The Polaroid Cube was also easy to operate. It is controlled by only one large button, making it easily accessible when it was in the rocket. The camera was held at the bottom of the dart's tail cone where a small hole allowed a dowel to be inserted to turn the camera on while on the launch pad. A photo of the Polaroid Cube is shown in Figure 5.

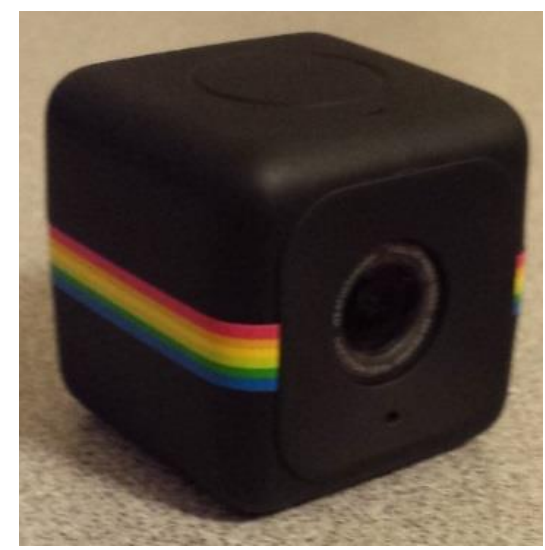

Figure 5: Polaroid Cube

In order to have video footage for the first stage of flight, the camera located in the tail cone of the dart had to film outside and downward on the booster section. To accomplish this, two designs where theorized. Using mirrors or fiber-optic cable, the camera would take in light from a hole in the transition on the booster and reflect it back to the camera in the tail cone of the dart. In theory, the fiber optic cable would be a simpler design however, imaging fiber is very expensive and the low cost end glow fiber purchased for testing did not have the cladding necessary for the total internal reflection making the image transmitted through the cable indistinguishable. For this 
reason the design using mirrors was chosen. One mirror was placed directly under the camera at a $45^{\circ}$ angle, and another mirror was placed outside of the rocket at a $45^{\circ}$ angle facing downward next to a hole in the transition. The mirrors were attached to the bulkhead used to support the dart during launch to provide a strong base during the rocket's flight.

1.7 Pressure relief. In order to deploy the parachutes, barometric pressure altimeters were implemented which require static pressure port holes to allow for the equalization of air pressure between the interior and exterior of the rocket. Without this equalization, the parachutes would not deploy at the right time and could deploy early or late depending whether the ports have the correct sizing. A general rule for the sizing of ports is to use a $1 / 4$ inch diameter hole (or hole area equivalent if several smaller holes are used) for each 100 cubic inches of volume in the electronics bay. Another general rule is the use of at least three holes that are spaced evenly around the circumference of the body of the rocket to negate the effect of crosswinds.

The diameter of the electronics bay is 2.14 inches and the inner length of the bay is 13.75 inches, yielding a volume of 49.5 cubic inches. A single port hole diameter was calculated to be 0.176 inches with an area of 0.024 square inches. Three holes were drilled into the electronics bay each with a diameter of 0.1015 inches, which is a \#38 drill bit. These holes were then spaced 120 degrees apart.

During the rocket's ascent the atmospheric pressure surrounding the rocket decreases. In order to relieve the pressure in other areas of the rocket, a quarter inch hole was drilled into each of the body sections of the rocket. Without these holes, the higher pressure inside the body sections of the rocket could cause early separation as well as early deployment of the parachutes.

1.9 Recovery. The dart and booster sections of the rocket used their own parachutes. The parachute's sizes were determined using online decent rate calculators and OpenRocket simulations to achieve a descent rate slow enough for the rocket to be recovered in a safe and flyable condition. The booster used a 44 inch SkyAngle parachute deployed with a time delay on the motor's ejection charge and the dart used a 36 inch parachute deployed at apogee using an altimeter. The dart had a descent rate of 13 feet per second and the booster had a descent rate of 11 feet per second once their respective parachutes opened. The parachutes are shown in Figure 6.

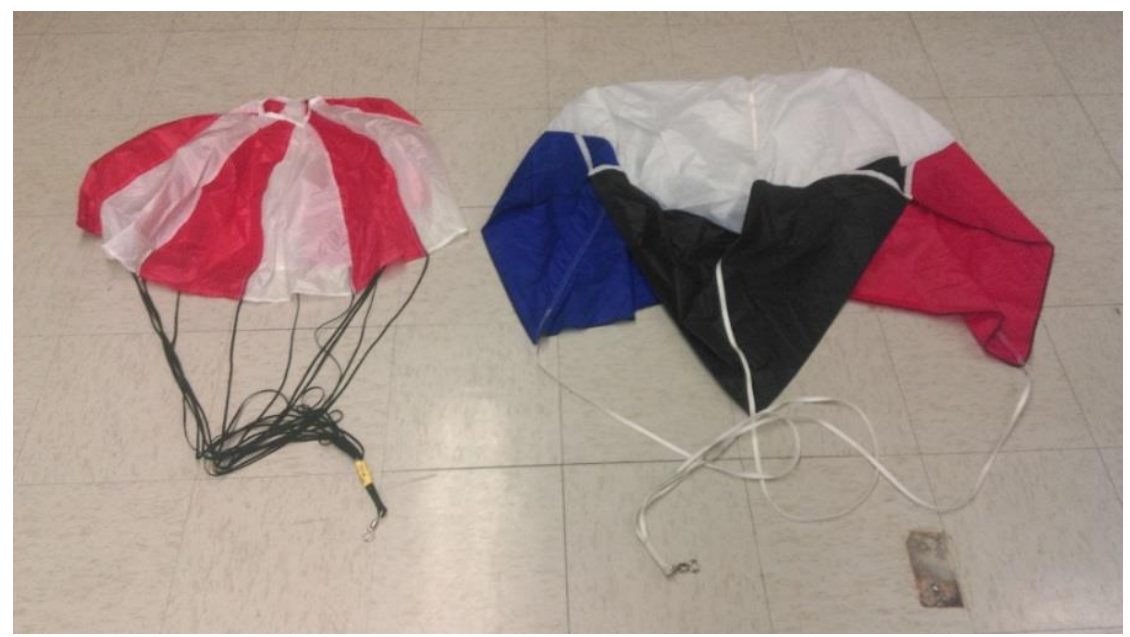

Figure 6: Dart and Booster Parachutes 


\subsection{Anticipated Performance}

The anticipated performance of the rocket was simulated using OpenRocket to estimate the performance of the rocket on launch day. The following sections detail these simulations.

2.1 OpenRocket. OpenRocket is a free, open source, software similar to RockSim. It is capable of calculating acceleration, velocity, and position data. This is done while accounting for variables including: elevation, wind speed, and the effects of individual components on performance. Also included in the program is the ability to construct full to-scale schematics of the rocket design. From this schematic the $\mathrm{CP}$ and $\mathrm{CG}$ can also be approximated.

OpenRocket was the main source used in designing the rocket. The rocket was modeled entirely in the program, providing a way to design and calculate proper lengths of body tubes, optimal fin and nosecone designs, rocket weights, acceptable locations of the $\mathrm{CP}$ and $\mathrm{CG}$, and drag coefficients. The weather parameters were monitored on launch day and entered into the simulations for better accuracy.

2.2 Flight predictions. The peak altitude, velocity and acceleration for the OpenRocket simulation on the day of the launch are shown in Table 3.

Table 3: Maximum Flight Predictions

\begin{tabular}{|l|c|}
\hline & OpenRocket \\
\hline Altitude (ft) & 2914 \\
\hline Velocity (ft/s) & 590 \\
\hline Acceleration(ft/s $\mathbf{2})$ & 679 \\
\hline
\end{tabular}

\subsection{Results}

An OpenRocket simulation was run to estimate flight performance of the rocket. Actual flight data was recorded using a Raven 3 flight data recorder provided by WSGC. In comparison, the flight of the rocket matched well with the estimates of the simulation. A comparison between predicted and measured results is shown in Table 4.

Table 4: Flight Performance Comparisons

\begin{tabular}{|l|c|c|c|}
\cline { 2 - 4 } \multicolumn{1}{c|}{} & Apogee (ft) & Maximum Velocity (ft/s) & Maximum Acceleration (ft/s $\mathbf{s}^{\mathbf{2}}$ ) \\
\hline OpenRocket & 2914 & 590 & 679 \\
\hline Actual & 3185 & 509 & 584 \\
\hline & \multicolumn{3}{|c|}{ Percent Error From Actual (\%) } \\
\hline OpenRocket & 9 & 14 & 14 \\
\hline
\end{tabular}

The predicted and actual acceleration data was compared graphically as well shown in Figure 7. 


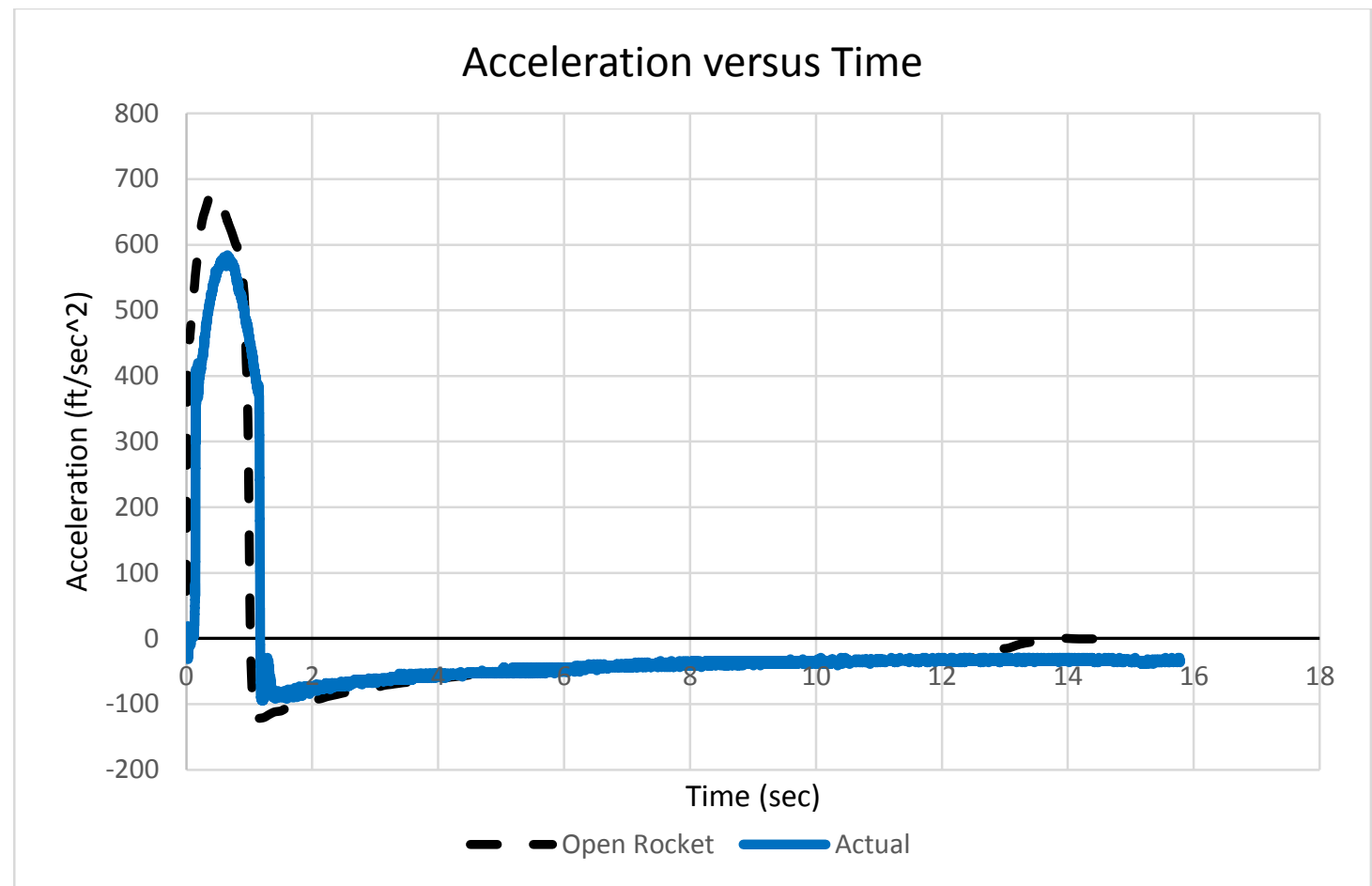

Figure 7: Comparison between Predicted and Actual Acceleration

The pitch and roll data recorded by the DAS is shown in Figure 8 .

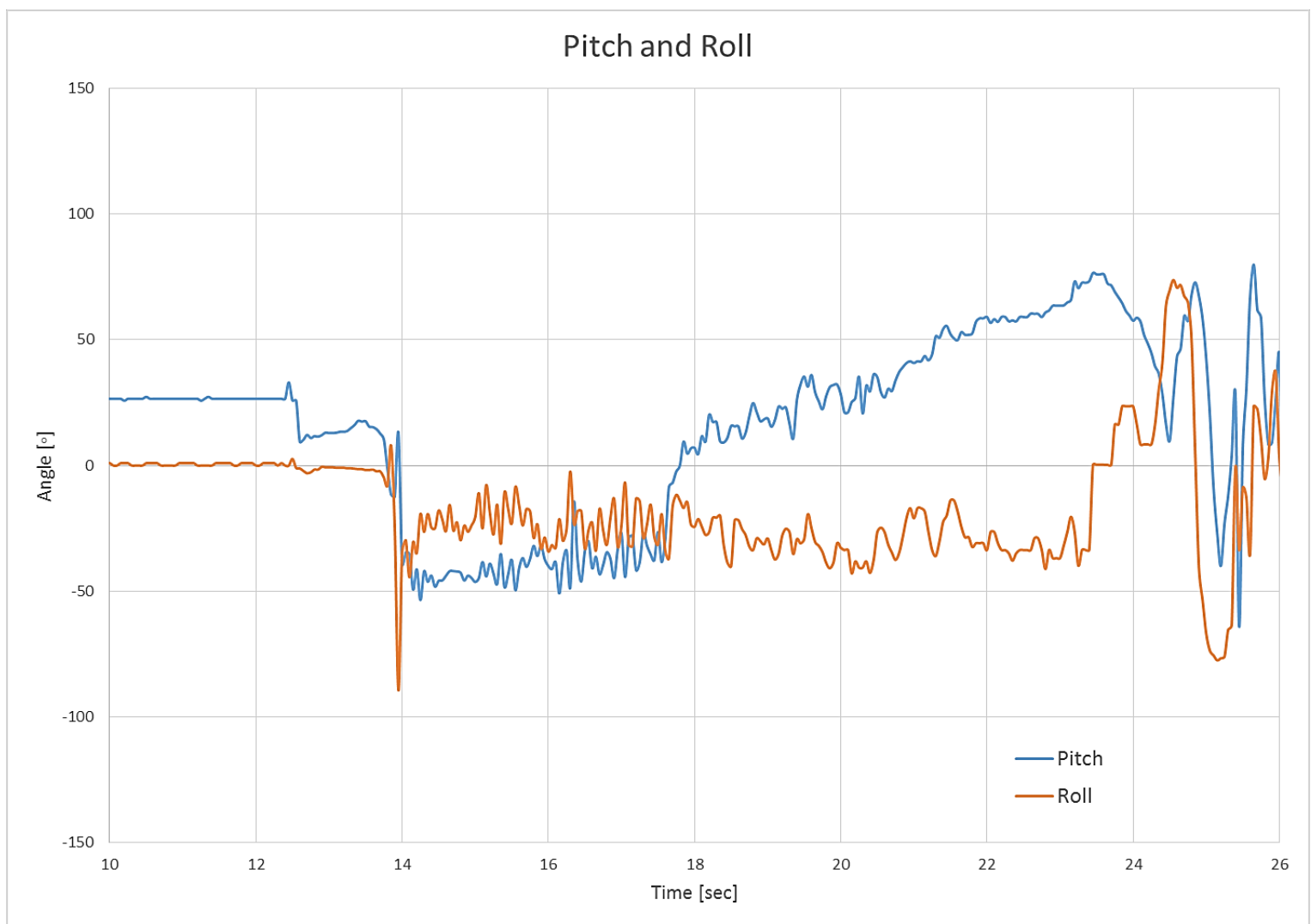

Figure 8: Pitch and Roll Data 
The plot shown in Figure 8 shows the pitch and roll of the dart during flight to apogee, calculated using the linear acceleration values from each axis on the dart. The pitch and roll data corresponds to the angle of the dart during launch, separation, apogee, and finally the black-powder charge ignition. The initial pitch offset is due to the angle of the launchpad, which was not perfectly vertical. The pitch plot shows the dart turning over as it approaches apogee while the roll plot shows that the dart experienced very little roll until the black-powder charge ignited. This is very close to what the rocket experienced during its flight.

The time to apogee was about 14 seconds. The rocket experienced a different acceleration curve than predicted shown in Figure 7. This could have been from the rocket's motor not producing the same thrust as the thrust curve data used in the OpenRocket simulation. The rocket overshot the predicted altitude of 2914 feet by 271 feet. The under prediction of the simulation was expected since the tail cone on the dart was not able to be modeled in the OpenRocket simulation. The tail cone reduces the base drag on the dart and thus gives the dart a higher apogee. With the expected under prediction of the simulation, a $14 \%$ error in the maximum acceleration and maximum velocity and a 9\% error in apogee shows the simulation was still accurate and thus was a good representation of the actual flight. The rocket performed mostly as planned. One system that was not properly implemented was the camera recording system. The camera was improperly turned on causing photos to be taken instead of video. This caused no flight video to be recorded.

\subsection{Conclusion}

The rocket was successfully recovered in a flyable condition in compliance with the competition rules. The software utilized for this design predicted the altitude of the rocket to a good margin given the uncertainties present in the launch and design. The camera system was improperly used showing that every system implemented on the rocket needs to be tested and re-tested in advance to ensure it will work as planned on launch day. The DAS recorded pitch and roll data that closely matched what occurred during the rocket's flight. Lessons learned through this design will be incorporated into future competitions by returning team members. A photo of the team is shown in Figure 9.

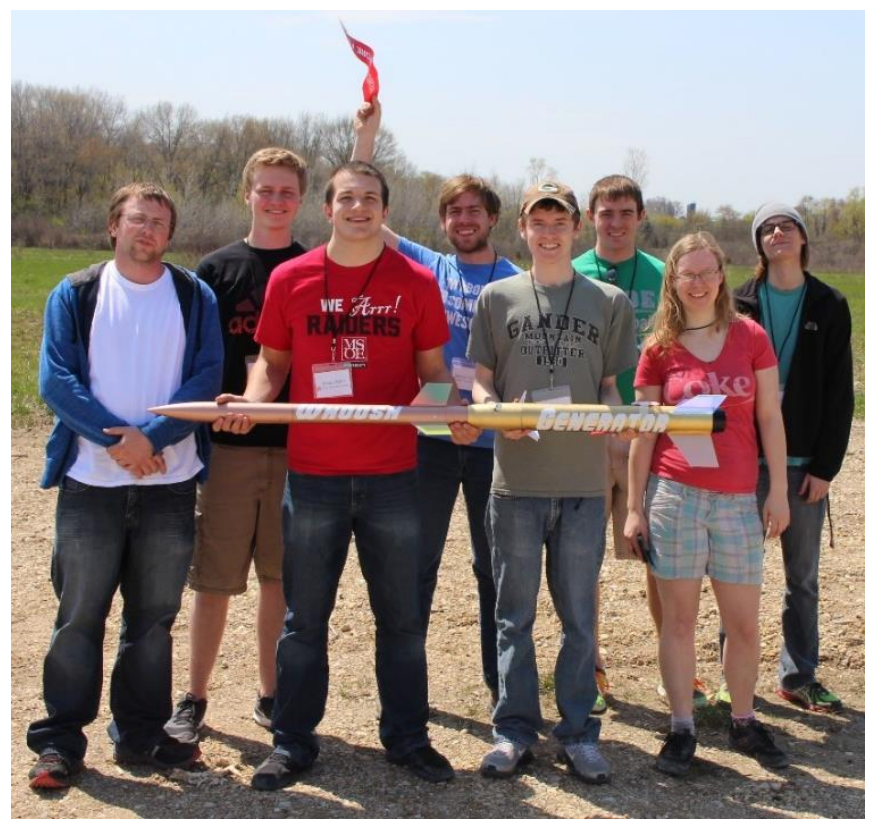

Figure 9: Team Whoosh Generator 2015 (not pictured, Lucas Ferrandoquielz) 